\title{
GENIE models and global fits of neutrino scattering data
}

Marco Roda* $*$

University of Liverpool, United Kingdom

E-mail: marco.rodaeliverpool.ac.uk

\begin{abstract}
Neutrino Monte Carlo generators are the single interface between theory and experiment and, as such, they are an essential ingredient in any attempt to connect neutrino data with the answers to fundamental physics questions. GENIE is the world's most widely-used neutrino Monte Carlo generator. Indeed, its physics model is employed by nearly all current and near future experiments. A key ingredient of the current effort to overhaul the GENIE phenomenological descriptions of neutrino scattering is to develop the capability to perform a global physics tuning using both the increasing body of neutrino scattering data and the vast complementary array of electron-nucleus and hadron-nucleus scattering data. GENIE already contains extensive curated data archives and can simulate the nuclear scattering of neutrinos, electrons and hadronic probes in the exact same physics framework. The vast set of GENIE comparisons to published data, along with GENIE machinery that allows the efficient incorporation of physics uncertainties in the GENIE predictions, will underlie the global tuning effort. A new collaboration with the Professor system authors used for Monte Carlo generator tuning for the Large Hadron Collider (LHC) experiments, provides the main algorithmic procedure for obtaining new physics tunes. In this talk we will outline the development of GENIE models for the simulation of CC $0 \pi$ events (a crucial event topology both for oscillation measurements and for probing nuclear dynamics) and present results of the first GENIE global fits of CC $0 \pi$ data.
\end{abstract}

The 19th International Workshop on Neutrinos from Accelerators-NUFACT2017

25-30 September, 2017

Uppsala University, Uppsala, Sweden

\footnotetext{
* Speaker.

${ }^{\dagger}$ on behalf of GENIE collaboration
} 


\section{Introduction}

In neutrino oscillation analyses, neutrino generators are by far the most common pathway to connect experimental observables and true neutrino energy. As a result, generators are responsible for the model dependencies which affect measurements of interesting physical quantities. Indeed, there is constant demand to improve the quality of predictions and produce results, out of the box, that are in good agreement with a large body of historical and recent measurements.

As neutrino oscillation physics is now in a precision era, systematic uncertainties have to be kept at the level few percent [1], requiring a campaign to improve the modeling of neutrino scattering. A key ingredient of this effort to overhaul the phenomenology is to develop the capability to perform a global physics tuning using both the increasing body of neutrino scattering data and the vast complementary array of electron-nucleus and hadron-nucleus scattering data.

There are a number of ways this might happen within a generator, but ideally this would be a global tuning including all the relevant data. Generators are the natural frameworks where global tuning should happen as they control the model implementations and they usually have extensive database to constrain their predictions. Also, predictions can come from non analytical models which makes the tuning impossible using only a theoretical framework. So far, tuning has been technically impossible due to the required computational effort.

In this context, the possibility of improvement strongly depends on the available data. Recently both quality and statistics of publicly available data are increasing together with our understanding of neutrino interaction in the few-GeV region [2]. Still, datasets suffer from the lack of a global analysis strategy neglecting obvious correlations, e.g. the one coming from extracting different datasets from the same neutrino beam.

\section{GENIE}

GENIE [3] is the most widely used neutrino generator and it is maintained by an international collaboration of about 20 members. Its main goal is to provide a state-of-art MC generator that can be used by the entire experimental neutrino community. As part of its core mission, the collaboration is actively involved in GENIE's model tuning against all sorts of publicly available data, including neutrinos, charged-leptons and hadron scattering data.

GENIE latest release is v2.12.6. This version contains a number of models for each relevant interaction at the few-GeV energy range, including most up-to-date developments. These can be combined in different ways to allow a number of different configurations.

\subsection{Comprehensive global configurations}

So far GENIE contained only one default comprehensive global configuration (CGC) which was supported and tuned by the collaboration. In practice, most experiments develop their own CGC. This makes the collaboration between experiments almost impossible if they are using different cross-section models. Starting from GENIE v3, there will be a number of CGC, each of them identified by its own unique code. These CGC are going to be well-tested and the collaboration guarantees that the single models won't have technical or physics issues when combined together 
in any given CGC. Even if GENIE v3 is yet to be released, some CGC are already designed and available on a dedicated GENIE's webpage: http://tunes.genie-mc.org/.

Two configurations are relevant for this talk: G16_01b and G16_02a. The first is an adiabatic update of the old GENIE default, including an empirical MEC model. The second is a CGC anchored on the latest theoretical models for CC $0 \pi$ and $1 \pi$ interactions. All the details can be found on GENIE's website.

\subsection{Comparisons}

As part of the GENIE v3 validation and deployment plan, each CGC will be characterized against the data available within the GENIE database. GENIE database consists of a separate software package that also provide the methods necessary to evaluate the right prediction for the data out of GENIE configuration. The database is constantly updated and contains some few tens of thousands points. This includes data from recent major neutrino experiments like MiniBooNE, T2K and MINERvA, but also electron scattering data, hadron-nucleon and hadron-nucleus events, historical neutrino cross-section measurements and several measurements of neutrino induced hadronic shower characteristics. Having in mind global tuning as a final goal, the system can store correlations between any set of measurements, even from different data releases.

Using this system, it's easy to compare the agreement of a model with respect to different datasets, even if data are flux integrated cross-sections from different neutrino fluxes. As a default the agreement is measured using $\chi^{2}$, including correlations if data contains it. The system allows to compare a number of models against different datasets to see which of the models is preferred by each dataset. If the preferred model is not the same for all datasets we will call this a tension. Interestingly, two of the most relevant $v_{\mu} \mathrm{CC} 0 \pi$ datasets are already showing tensions. Even though both datasets point toward models that include 2p2h interaction, MiniBooNE [4] data prefers G16_02a while T2K [5] favors G16_01b.

\section{Tuning}

The conceptual requirements for a global tuning are simple: the datasets, a way to provide a prediction against the data as a function of some parameters and a metric of the agreement between predictions and data. All of these are granted by GENIE's comparisons, what is missing is a minimization algorithm. The latter is an old problem in high energy physics given the high CPU time required to obtain a prediction for each point of the parameter space, which in case of GENIE takes hours.

The solution was found in the Professor suite [6]. Fundamentally, the idea is to reduce the expensive process of brute-force tuning allowing for massive parallelisation by use of a deterministic parameterisation of the generator's response to changes in the steering parameters. In addition to the obvious time gain, this makes possible the tuning of all parameters of GENIE, including those which are not reweightable using the reweigh package [7].

\section{1 $0 \pi$ global fit}

The $0 \pi$ global tuning was performed using both neutrino and antineutrino data from different experiments. The complete list of the datasets is: MiniBooNE CCQE neutrino [4] and anti-neutrino 
Table 1: G16_01b parameters tuning results. Whenever an uncertainty is reported in the nominal value, that is the uncertainty quoted in the reweight package.

\begin{tabular}{r|c|c|c} 
Parameter & Range & Best fit & Nominal \\
\hline QEL- $M_{A}\left(\mathrm{GeV} / c^{2}\right)$ & {$[0.7 ; 1.8]$} & $1.17 \pm 0.03$ & $0.99 \pm 0.01$ \\
QEL-CC-XSecScale & {$[0.8 ; 1.2]$} & $0.92 \pm 0.02$ & 1 \\
MEC-FracCCQE & {$[0 ; 1]$} & $0.55 \pm 0.06$ & 0.45 \\
RES-CC-XSecScale & {$[0.5 ; 1.5]$} & $1.02 \pm 0.07$ & 1 \\
FSI-PionMFP-Scale & {$[0.6 ; 1.4]$} & $0.86 \pm 0.04$ & $1.0 \pm 0.2$ \\
FSI-PionAbs-Scale & {$[0.4 ; 1.6]$} & $0.76 \pm 0.09$ & $1.0 \pm 0.3$
\end{tabular}

Table 2: G16_02a parameters tuning results. Whenever an uncertainty is reported in the nominal value, that is the uncertainty quoted in the reweight package.

\begin{tabular}{r|c|c|c} 
Parameter & Range & Best fit & Nominal \\
\hline QEL- $M_{A}\left(\mathrm{GeV} / c^{2}\right)$ & {$[0.7 ; 1.8]$} & $1.00 \pm 0.03$ & $0.99 \pm 0.01$ \\
QEL-CC-XSecScale & {$[0.8 ; 1.2]$} & $0.91 \pm 0.02$ & 1 \\
MEC-CC-XSecScale & {$[0.7 ; 1.3]$} & $1.18 \pm 0.02$ & 1 \\
RES-CC-XSecScale & {$[0.5 ; 1.5]$} & $1.01 \pm 0.04$ & 1 \\
FSI-PionMFP-Scale & {$[0.6 ; 1.4]$} & $1.17 \pm 0.04$ & $1.0 \pm 0.2$ \\
FSI-PionAbs-Scale & {$[0.4 ; 1.6]$} & $1.02 \pm 0.09$ & $1.0 \pm 0.3$
\end{tabular}

[8] data, MINERvA CCQE neutrino [9] and anti-neutrino data [10] and neutrino CC $0 \pi$ data from T2K ND280 [5]. Even though these are all flux integrated differential cross-sections data, format and quality of the dataset are different. MiniBooNE's data contains the the majority of the points, but they lack of a full covariance matrix, while T2K and MINERvA data contains a full covariance matrix for each histogram. It has to be noticed that MINERvA experiment released a full covariance matrix between neutrino and antineutrino data, but that was not used in this tuning.

Two different models were tuned: G16_01b and G16_02a. Conceptually, the same parameters were tuned: QE axial mass, a rescaling factor of CC QE cross-section, a rescaling factor of CC $2 \mathrm{p} 2 \mathrm{~h}$ cross-section, a rescaling factor of the total CC RES cross-section and rescaling factors for Pion Mean Free Path (MFP) and Pion Absoption. Because of the different implementations, the $2 \mathrm{p} 2 \mathrm{~h}$ rescaling factor is different for the two models, see Tables 1 and 2.

Rescaling nuisance parameters for GENIE predictions were included into the fit for MiniBooNE data, one for neutrinos and one for anti-neutrinos data. This was done to take into account the missing correlation matrix and it is also an approach suggested by Nieves et al. in [11]. Both the parameters range from 0.7 to 1.3 and, interestingly, they both converge to 1.1 , regardless of the dataset or the model. It is remarkable that the rescaling parameter obtained by Nieves is $1.09 \pm 0.04$. This is also consistent with the MiniBooNE flux error estimation [12]. In the fit a prior on QELCC-XSecScale and RES-CC-XSecScale was inserted: for both it is a gaussian with center 1 and sigma 0.1 .

\subsection{Results and discussion}

The result of the fit can be seen in Tables 1 and 2 for both models which were tuned. In terms of physics outputs there are common patterns that are relevant. First of all, $M_{A}$ is reasonably low, and, in case of G16_02a, it is in perfect agreement with the value extracted on single nucleons in [13]. This is an independent check of a parameter that has to be extracted from data and for that 
reason no prior was associated with this parameter. The second observation is that in both cases, the QEL contribution is reduced by $10 \%$ and the $2 \mathrm{p} 2 \mathrm{~h}$ contribution is increased by $20 \%$. We don't attempt to give any phenomenological explanation of this result.

Another point of the discussion is the agreement of the fitted model against the datasets. The output parameters given by the minimization using the polynomial interpolation is used to created a full GENIE production. The total $\chi^{2}$ dropped in both cases and that means the fitting machinery is working, see Figure 1. Because of the larger number of points, the fit is driven by MiniBooNE datasets which are the only one that can be fitted separately giving physical results. This is quite unsatisfactory given that the missing covariance matrix makes these data the one with lowest quality. It is important to point out that the total $\chi^{2}$ dropped, but the agreement with T2K ND280 $0 \pi$ dataset either remain unchanged (G16_02a) or decreased (G16_01b). This should not surprise as tensions between MiniBooNE and T2K ND280 were pointed out in previous sections.
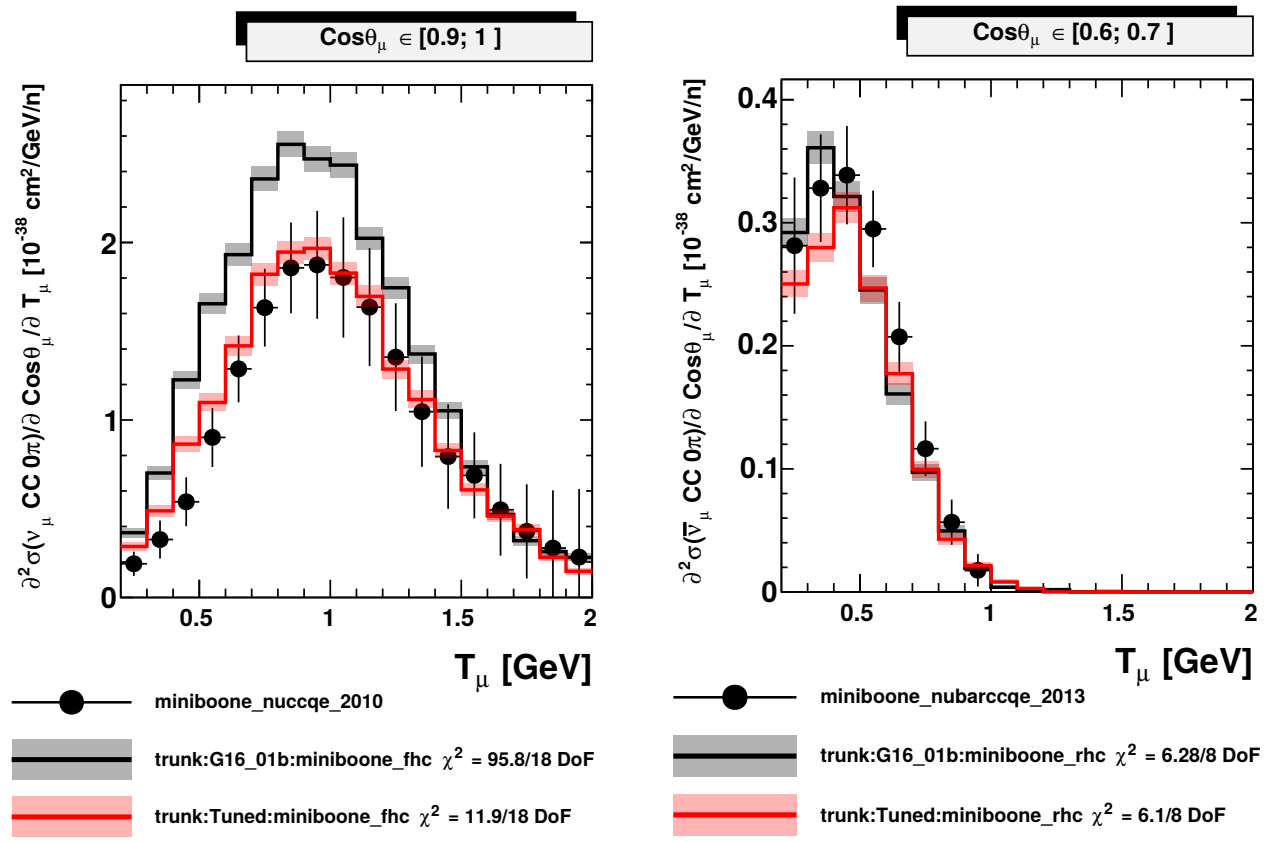

Figure 1: Examples of tuned G16_01b (red) against nominal parameter configuration (black) compared to slices double differential cross-sections data from MiniBooNE (black dots). Neutrino (left) and antineutrino (right) data. The reported $\chi^{2}$ are evaluated only using the data in the slices. The color bands around the predictions lines are the statistical error due to the MC sample used to evaluate the model.

\section{Conclusions}

Genie is undergoing a big renovating process. Most of the improvements rely on a rich and yet growing database of publicly available data about neutrino interactions, charged lepton scattering data, and hadronic data.

The new models available in GENIE are deployed in CGCs, which are characterized against the internal GENIE database. Each of these models has a number of parameters that can be optimized by means of global fits, therefore a dedicated machinery based numerical assistant called 
Professor was set up. The machinery was validated through a $0 \pi$ data tuning, effectively opening a new branch of analysis within the GENIE suite. GENIE collaboration is already planning a number of tuning: re-tune of hadronization model, re-tune of (anti-)neutrino cross-sections on free nucleons revisiting the strategy for transitioning from resonances to DIS, global tuning of $1 \pi$ data and a combined global fit of $0 \pi$ and $1 \pi$ data.

What can be done with this new machinery strongly depends on the quality of the available data. In a few years new experiments like SBND will start providing data with an unprecedented combination of accuracy and statistics. We look forward to a promise collaboration between generators experiments and model developers.

\section{References}

[1] DUNE collaboration, R. Acciarri et al., Long-Baseline Neutrino Facility (LBNF) and Deep Underground Neutrino Experiment (DUNE), 1512.06148.

[2] T. Katori and M. Martini, Neutrino-nucleus cross sections for oscillation experiments, Journal of Physics G: Nuclear and Particle Physics (2017), [1611.07770].

[3] C. Andreopoulos et al., The GENIE Neutrino Monte Carlo Generator, Nucl. Instrum. Meth. A614 (2010) 87-104, [0 905 .2517].

[4] MiniBoonE collaboration, A. A. Aguilar-Arevalo et al., First Measurement of the Muon Neutrino Charged Current Quasielastic Double Differential Cross Section, Phys. Rev. D81 (2010) 092005, [1002.2680].

[5] T2K collaboration, K. Abe et al., Measurement of double-differential muon neutrino charged-current interactions on $\mathrm{C}_{8} \mathrm{H}_{8}$ without pions in the final state using the T2K off-axis beam, Phys. Rev. D93 (2016) 112012, [1602.03652].

[6] A. Buckley, H. Hoeth, H. Lacker, H. Schulz and J. E. von Seggern, Systematic event generator tuning for the LHC, Eur. Phys. J. C65 (2010) 331-357, [0 907 . 2973].

[7] C. Andreopoulos, C. Barry, S. Dytman, H. Gallagher, T. Golan, R. Hatcher et al., The GENIE Neutrino Monte Carlo Generator: Physics and User Manual, 1510.05494.

[8] MiniBoonE collaboration, A. A. Aguilar-Arevalo et al., First measurement of the muon antineutrino double-differential charged-current quasielastic cross section, Phys. Rev. D88 (2013) 032001, [1301.7067].

[9] MINERvA collaboration, G. A. Fiorentini et al., Measurement of Muon Neutrino Quasielastic Scattering on a Hydrocarbon Target at $E_{v} \sim 3.5 \mathrm{GeV}$, Phys. Rev. Lett. 111 (2013) 022502, [1305.2243].

[10] MINERvA collaboration, L. Fields et al., Measurement of Muon Antineutrino Quasielastic Scattering on a Hydrocarbon Target at $E_{v} \sim 3.5 \mathrm{GeV}$, Phys. Rev. Lett. 111 (2013) 022501, [1305.2234].

[11] J. Nieves, I. Ruiz Simo and M. J. Vicente Vacas, The nucleon axial mass and the MiniBooNE Quasielastic Neutrino-Nucleus Scattering problem, Phys. Lett. B707 (2012) 72-75, [1106. 5374 ].

[12] MiniBoone collaboration, A. A. Aguilar-Arevalo et al., The Neutrino Flux prediction at MiniBooNE, Phys. Rev. D79 (2009) 072002, [0 806.1449 ].

[13] K. S. Kuzmin, V. V. Lyubushkin and V. A. Naumov, Quasielastic axial-vector mass from experiments on neutrino-nucleus scattering, Eur. Phys. J. C54 (2008) 517-538, [0712 .4384]. 